OPEN ACCESS

Edited by:

Oliver Wirths,

University Medical

Center Göttingen, Germany

Reviewed by:

Jessica Elaine Young,

University of Washington,

United States

Shreyasi Chatteriee,

University of Southampton,

United Kingdom

*Correspondence:

Lian Li

lilian1222@hotmail.com

Received: 07 November 2018 Accepted: 02 August 2019

Published: 22 August 2019

Citation:

Liu J and Li L (2019) Targeting

Autophagy for the Treatment of Alzheimer's Disease: Challenges and

Opportunities.

Front. Mol. Neurosci. 12:203. doi: 10.3389/fnmol.2019.00203

\section{Targeting Autophagy for the Treatment of Alzheimer's Disease: Challenges and Opportunities}

\author{
Jie Liu and Lian Li* \\ Translational Center for Stem Cell Research, Stem Cell Research Center, Tongji Hospital, Tongji University School \\ of Medicine, Shanghai, China
}

Alzheimer's disease (AD) is the most common type of dementia which characterized by a progressive loss of memory and cognitive function due to degeneration of synapses and axons. Currently, there is no cure for AD. Deposition of extracellular amyloid- $\beta(A \beta)$ plaques and intracellular tau neurofibrillary tangles (NFTs) are two hallmark pathologic changes in the brains of Alzheimer's patients. Autophagy is the major mechanism in cells responsible for removing protein aggregates. Accumulation of immature autophagic vacuoles (AVs) in dystrophic neurites of Alzheimer patients' brains suggests that autophagy process is disrupted. Till now, it is far from clear what role autophagy plays in $A D$, a causative role, a protective role, or just a consequence of the disease process itself. To design more effective therapeutic strategies towards this devastating disorder, it is essential to understand the exact role of autophagy played during different stages of AD.

Keywords: Alzheimer's disease, autophagy, autophagic vesicle, amyloid- $\beta$ plaque, tau neurofibrillary tangle, synapse, axon

\section{INTRODUCTION}

Alzheimer's disease $(\mathrm{AD})$ is a progressive neurodegenerative disorder and the most common form of dementia in the elderly, which is characterized by a progressive deficiency in memory and cognitive functions (Scheltens et al., 2016). More than 50 million people are affected by AD worldwide (Hodson, 2018). Autosomal dominant mutations in amyloid precursor protein (APP), presenilin 1 (PS1), or presenilin 2 (PS2) cause early-onset familial AD (fAD). Whereas the vast majority ( $>95 \%)$ of $\mathrm{AD}$ cases develop sporadically without a clear genetic component or etiology, which are known as sporadic AD (sAD). Additionally, as the main risk factor for $\mathrm{AD}$ is aging, $\mathrm{AD}$ is predicted to become a major socioeconomic burden in the near future with the average life expectancy on the rise (Xia et al., 2018). Deposition of extracellular amyloid- $\beta$ plaques ( $A \beta$, aggregated $\beta$-amyloid peptide) and intraneuronal tau neurofibrillary tangles (NFTs, aggregated hyperphosphorylated tau protein) in specific brain regions are two major lesions of this devastating pathology (Blennow et al., 2006).

Abbreviations: AD, Alzheimer's disease; APP, amyloid precursor protein; PS1, presenilin 1; PS2, presenilin 2; fAD, familial $\mathrm{AD}$; sAD, sporadic $\mathrm{AD} ; \mathrm{A} \beta$, amyloid- $\beta$; NFTs, neurofibrillary tangles; Atg, autophagy-related genes; $\mathrm{AVs}$, autophagic vacuoles; apoE4, apolipoprotein E4; LC3, microtubule-associated protein 1A/1B-light chain 3; mTOR, mammalian target of rapamycin; Bcl2, B-cell lymphoma 2; TFEB, transcription factor EB; HD, Huntington's disease; PD, Parkinson's disease. 
Through proteolytic cleavage by $\beta$ - and $\gamma$-secretase sequentially, $\mathrm{A} \beta$ is generated from APP, and then secreted at the plasma membrane (Vassar et al., 1999; De Strooper et al., 2012). In aggressive early-onset fAD patients, mutations of APP or $\gamma$-secretase-associated PS1 and PS2 were identified, which strongly links $\mathrm{A} \beta$ to $\mathrm{AD}$. The amyloid cascade hypothesis was formed based on this strong causative relationship between $A \beta$ and AD (Hardy and Higgins, 1992; Karran et al., 2011), which assumes that the deposition of $A \beta$ peptide in the brain is a central event in $\mathrm{AD}$ pathogenesis and predicts that $\mathrm{A} \beta$ accumulation precedes the NFTs formation. Clinically, the extent of tau NFTs formation correlates closely with the cognitive dysfunction of $\mathrm{AD}$ to a greater degree than does $A \beta$ plaque load (Nelson et al., 2012). However, the etiology mechanisms underlying these pathological changes and progressive loss of synapses and neurons in $\mathrm{AD}$ are not clear yet.

Macroautophagy [hereafter referred to as autophagy, see review by Cuervo and Wong (2014) for chaperone-mediated autophagy] is a conserved process for clearing of long-lived proteins, protein aggregates, dysfunctional cellular organelles and invaded pathogens, which is an essential mechanism for maintenance of cellular homeostasis (Dikic and Elazar, 2018). Recently, it has become apparent that autophagy plays a central role in development, aging, and neurodegeneration. In addition, levels of autophagy are exquisitely regulated in different cells. Homeostasis and survival of neurons depend on the essential role of basal autophagy. The post-mitotic nature of neurons predisposes them to the deposition of misfolded proteins and damaged organelles which otherwise could be diluted through cell division in replicating cells (Metaxakis et al., 2018). Mice deficiency of the essential autophagy-related gene 5 (Atg5) or Atg7 specifically in the central nervous system exhibit progressive neuronal degeneration with abnormal intracellular proteins accumulated and a large number of aggregates and inclusions developed (Hara et al., 2006; Komatsu et al., 2006). Synapses are regions of quick protein turnover with high energy demand. Coordinated protein synthesis and degradation are indispensable for the morphological and functional modifications of synapses (Nikoletopoulou and Tavernarakis, 2018). It is now widely accepted that neuronal autophagy is essential for the synapse plasticity, which is required for learning and memory that is impaired in AD (Bingol and Sheng, 2011; Hernandez et al., 2012). Accumulating evidence also supports that, for the maintenance of local axon homeostasis and protection against axonal degeneration under stress conditions, normal function of autophagy is particularly important. In the distal region of the axon, Hollenbeck observed real-time autophagosome formation, which suggested autophagosome biogenesis in the axons locally (Hollenbeck, 1993). By employing live imaging, recent studies clearly showed that autophagosomes are initially produced in the distal terminals of axons, then transported to the soma retrogradely, and finally ended in completing the degradation of its contents by fusion with lysosomes (Yue, 2007; Maday et al., 2012). All of the above studies indicated that during the neurite extension and maintenance process autophagy was a key mechanism for shaping the structures of neurite and growth-cone, and was essential for neural plasticity.
In the central nervous system, suppression of basal autophagy causes severe axonal swelling and atrophy, which leads to neurodegeneration eventually (Hara et al., 2006; Komatsu et al., 2006).

However, several works confirmed the scarcity of autophagosomes in healthy neurons (Mizushima et al., 2004; Nixon et al., 2005; Boland et al., 2008), which may due to high efficiency of autophagosome clearance in neurons (Boland and Nixon, 2006; Boland et al., 2008). In addition, researches show that autophagy is more efficient in young neurons than in old ones (Boland et al., 2008), as autophagy-related proteins such as Atg5, Atg7, and beclin-1 will decline with aging (Shibata et al., 2006; Lipinski et al., 2010), which probably contributes to the late onset of several neurodegenerative diseases including $\mathrm{AD}$ (Harris and Rubinsztein, 2011).

\section{MALFUNCTION OF AUTOPHAGY IN AD}

A substantial amount of evidence supports that autophagy dysregulation occurs in both AD patients and animal models. As early as 1967, Suzuki found that, in AD patient brains, there were a large amount of abnormal subcellular vesicles and aggregated tau protein accumulated in the swollen or dystrophic neuritis (Suzuki and Terry, 1967). The identity of these vesicles was unknown at that time. In 2005, by using immunogold labeling and electron microscopy, Nixon's group found that these vesicles accumulated in dystrophic neurites in AD brains were immature autophagic vacuoles (AVs; Nixon et al., 2005). Data from PS1/APP double transgenic mice also showed that large amount of AVs accumulated in neuronal dendrites and soma before $A \beta$ plaques appeared when compared to age-matched controls ( $\mathrm{Yu}$ et al., 2005). In hippocampal neurons of AD mice, far before the synaptic and neuronal loss, abnormal accumulation of immature AVs in axon was observed (Tomiyama et al., 2010; Sanchez-Varo et al., 2012). In several other animal models of AD including TgCRND8 mice over-expressing mutant human APP695 and $\mathrm{APP}_{\mathrm{SWE}} / \mathrm{PS}_{\mathrm{M} 146 \mathrm{~L}}$, the abnormal accumulation of AVs has also been observed (Cataldo et al., 2004; Yang et al., 2011a). Tau aggregates are degraded through autophagy pathway (Wang and Mandelkow, 2012; Ji et al., 2017). Autophagic gridlock also contributes to the development of AD-like tauopathy (Bakhoum et al., 2014). The abundance of $\mathrm{AVs}$ in the brains of $\mathrm{AD}$ animal models and $\mathrm{AD}$ patients is in sharp contrast to the rarely-observed AVs in normal brains, which suggests that the accumulation of pathogenic proteins such as $A \beta$ and tau in $\mathrm{AD}$ may be caused by defective autophagy-lysosome proteolysis pathway (Cataldo et al., 2004; Yang et al., 2011a).

So far, three genes, APP, PS-1, and PS-2, have been identified as causative genes for fAD (Tang and Gershon, 2003). Research has found wild type PS1 but not mutation forms, by regulating the distribution of $\mathrm{v}$-ATPase subunit V0a1 onto lysosome, is crucial for lysosome acidification and thus contributes to the regulation of autophagy-lysosome degradation system in a $\gamma$-secretase-independent way (Lee et al., 2010). As a major genetic risk factor for $\mathrm{sAD}$, apolipoprotein $\mathrm{E} 4$ (apoE4) has been found to induce malfunction of autophagy. A $\beta 42$ in lysosome were significantly elevated in ApoE4 transgenic mice, which 
finally led to neuronal death in the hippocampus (Belinson et al., 2008). In addition, other study found that in Neuro-2a cells ApoE4 potentiates leakage from lysosome and enhances A $\beta$ peptide-induced apoptosis (Ji et al., 2006). Progressive accumulation of AVs and lysosomal deficits in the brain has been widely recognized as another hallmark of AD (Nixon and Yang, 2011), though it is still a debate whether autophagy dysfunction is the result or the cause of AD (Shin et al., 2014; Peric and Annaert, 2015). Furthermore, gender difference may have an influence on the malfunction of auto-lysosome system (Congdon, 2018). In general, accumulating evidence seems to indicate that in the early stages of $\mathrm{AD}$ development autophagy has a protective role, whereas it appears to potentiate neuronal degeneration in the more advanced stages.

\section{MECHANISMS UNDERLYING AUTOPHAGY IMPAIRMENT IN AD}

The abnormal accumulation of autophagosomes in neurons of $\mathrm{AD}$ brain constituted the first sign of autophagy deficits. But, it is not fully understood what is the exact mechanism underlying dysfunction of autophagy in AD (Liang and Jia, 2014). The build-up of AVs in neurodegenerative diseases may reflect enhanced autophagy induction, impaired later lysosomal degradation steps in the autophagic pathway, or a lower rate of autophagy initiation combined with insufficient lysosome fusion and digestion (Barnett and Brewer, 2011). In general, the field is still at odds over which stage or stages of the autophagiclysosomal pathway is dysfunctional in AD.

\section{Altered Autophagy Initiation in AD}

Although autophagosomes are numerous in brains of the $\mathrm{PS}_{M 146 L} / \mathrm{APP}_{751 S L}$ mouse and in brains of $\mathrm{AD}$ patients, it does not necessarily indicate that autophagy induction is upregulated. Actually, the expression of beclin-1 which is an essential protein for autophagy initiation is decreased in brains of $\mathrm{AD}$ patients when compared with that of healthy individuals (Liang et al., 1999; Pickford et al., 2008). The loss of beclin-1 is believed to be caused by the increased activity of caspase 3 , as this enzyme-mediated cleavage of beclin-1 occurs in brains of $\mathrm{AD}$ patients (Rohn et al., 2011). In an APP transgenic mouse model with beclin-1 deletion, the basal level of autophagy is disrupted, and intracellular $A \beta$ accumulation increased (Pickford et al., 2008). Protein p62 is an autophagic cargo receptor, which was shown to bind directly to microtubule-associated protein $1 \mathrm{~A} / 1 \mathrm{~B}-$ light chain 3 (LC3; Pankiv et al., 2007). In a triple transgenic mouse model of AD (3xTg-AD), Du et al. (2009a,b) observed a significant decrease in p62 expression. On the contrary, a genome-wide research indicated that autophagy is up-regulated specifically in $\mathrm{AD}$, due both to the transcriptional up-regulation of positive regulators of autophagy and to the reactive oxygen species-dependent activation of a critical kinase for the initiation of autophagy, the type III PI3 kinase (Lipinski et al., 2010). Bordi et al. (2016) reported that autophagy flux increased in CA1 neurons of Alzheimer hippocampus as indicated by striking upregulation of Atgs, increases in autophagosome formation and lysosomal biogenesis beginning at early $\mathrm{AD}$ stages. As controversy is evident, more effort should be made in this area to assess real-time autophagy activity at different stages of $\mathrm{AD}$ pathogenesis. Autophagy may be regulated differentially in the early stage and late stage of AD. In this regard, developing reliable in vivo autophagy flux assay methodology should be a priority for the field.

\section{Disrupted Transportation of Autophagosome}

In normal neurites, for final lysosomal degradation, immature AVs are transported retrogradely from distal axon terminals towards the soma. In the $\mathrm{AD}$ brain, their transportation might be impeded as suggested by the significant build-up of AVs within dystrophic neuritis (Nixon, 2007). In mouse and cell models of $\mathrm{AD}$, it has also been reported that the transportation of autophagy-related compartments is selective defected (Nixon and Yang, 2011). Inhibiting the delivery of autophagosomes to lysosomes induces a rapid AVs accumulation in neurites, with very similar morphology to what has seen in the $\mathrm{AD}$ brain, which further suggests that defective axonal transportation of AVs may play a role in AD pathogenesis (Boland et al., 2008).

In the central nervous system, tau protein is mainly found in neurons, where it primarily localizes in axons and to a much lesser extent in dendrites and neuronal soma (Binder et al., 1985). Tau is effectively degraded through the autophagy pathway and regulates autophagy in reverse (Caballero et al., 2018). Several studies have shown that autophagy-lysosome system impairment leads to the formation of tau oligomer and insoluble aggregate, whereas their formation can be significantly alleviated through the induction of autophagy (Hamano et al., 2008; Congdon et al., 2012). In addition, autophagy dysfunction can be a result of tau hyperphosphorylation as tau modifications can provoke lysosomal aberrations (Lim et al., 2001; Lin et al., 2003). Tau is critical for autophagosome retrograde trafficking and maturation to fuse with lysosome through facilitating the assembly and stabilization of microtubule (Dixit et al., 2008). Importantly, in AD models, tau may gain a toxic function as tau deficiency is largely protective against $A \beta$ toxicity, which suggests that $A \beta$-mediated neurotoxicity seems to require tau in $\mathrm{AD}$ (Roberson et al., 2007; Ittner et al., 2010). However, other studies offer an opposite point of view that the real causative factor of axonal dysfunction is the abnormality of lysosomal proteases (Xie et al., 2015). To clarify the molecular defects that underlie the AVs transportation failure in $\mathrm{AD}$, more studies are required to identify the role of each defect.

\section{Defective Lysosomal Fusion/Degradation in $A D$}

There are reports show that PS1 is critical for the acidification of lysosome and the fusion of the autophagosome with the lysosome. Abnormal accumulation of AVs have been identified in fibroblasts derived from patients with fAD-linked PS1 mutations, where markedly impairment of the turnover of long-lived proteins is detected (Esselens et al., 2004; Lee et al., 2010, 2015; Neely et al., 2011). Others believe that lysosomal calcium homeostasis defects, but not proton pump defects, causes 
endo-lysosomal dysfunction in PS1-deficient cells (Coen et al., 2012; Zhang et al., 2012). Anyway, supported by accumulating evidence, a critical role of lysosomal proteolytic failure has been suggested in the development of neurodegeneration in $\mathrm{AD}$ (Colacurcio et al., 2018). In AD brain, the AVs accumulated are electron-dense autolysosomes and autophagosomes which are filled with undigested or incompletely-digested "waste" proteins (Nixon et al., 2005). Abnormally high levels of $A \beta$, ubiquitinated proteins, and LC3-II are presented in the lysosome and AVs fractions isolated from the brains of TgCRND8 mice (Yang et al., 2011b). The morphology of accumulated AVs in brains of a transgenic mouse model of $\mathrm{AD}$ and $\mathrm{AD}$ patients is very similar to that induced by blockage of lysosomal proteolysis with specific cathepsin deletion (Koike et al., 2000; Boland et al., 2008). In neurons, selectively blocking of cathepsin-mediated proteolysis within autolysosomes using inhibitors of cysteine-protease or aspartyl-protease also leads to a marked accumulation of AVs with electron-dense doublemembrane which contained incompletely degraded LC3-II (Boland et al., 2008). The above evidence strongly indicates the principal mechanism underlying autophagic dysfunction in $\mathrm{AD}$ may be the disruption of substrate proteolysis within the autolysosome.

On the other hand, works done by others suggests that APP metabolites may also accumulate in cells through inhibition of autophagy before fusion with lysosome, such as genetic deletion of ATG7 or ATG5, which indicating that AD-like pathology may arise by perturbation at any step along the autophagylysosome pathway (Tian et al., 2011). In addition, autophagy plays an important role in $A \beta$ metabolism. First, autophagy is believed to be another major $A \beta$ clearance pathway (Nilsson and Saido, 2014) along with $A \beta$ degradation enzymes (Miners et al., 2008). Second, under physiological conditions, the autophagylysosome pathway is important for the degradation of $A \beta$; whereas under pathological condition or during the process of aging, it is demonstrated the autophagy-lysosome system is a novel pathway for the production of $\mathrm{A} \beta$ (Yu et al., 2005). Third, the secretion of $A \beta$ is also mediated by autophagy. Recent findings demonstrate that extracellular release of $\mathrm{A} \beta$ through the autophagy pathway. Genetic deletion of essential autophagy component leads to inhibition of $\mathrm{A} \beta$ secretion and reduced intracellular accumulation of $A \beta$, which further exacerbated neurodegeneration (Nilsson et al., 2013). In return, $A \beta$ could also regulate autophagy. In neurons, $A \beta$ could directly induce autophagy (Hung et al., 2009), disrupt autolysosomal membrane physical integrity, and impair substrate degradation in the lysosome (Ling et al., 2009). It seems that a pro-survival role is played initially by neuronal autophagy induced by $\mathrm{A} \beta 42$, which is switched to a pro-death role in a time-dependent manner.

\section{AUTOPHAGY MODULATION FOR AD THERAPY}

\section{Promoting Autophagy Induction}

By integrating many signaling cascades in the cell, the mammalian target of rapamycin (mTOR) is a well-established key pathway that senses nutrient and regulates cell metabolism (Noda and Ohsumi, 1998; Corradetti and Guan, 2006; Pei and Hugon, 2008). Genetic reduction of mTOR signaling in Tg2576 mice brain enhanced autophagy induction (Figure 1) and restored normal hippocampal gene expression signature, and results in a reduced deposit of $\mathrm{A} \beta$ and alleviated memory deficits (Caccamo et al., 2014). Signaling through mTOR regulates tau homeostasis (Tang et al., 2013). Pharmacologically reducing mTOR signaling with rapamycin ameliorated tau pathology (Caccamo et al., 2013). Long-term inhibition of mTOR by rapamycin or latrepirdine also prevents AD-like cognitive deficits and lowers A $\beta 42$ level, reduces amyloid plaques and tau NFTs (McGowan et al., 2005; Caccamo et al.,

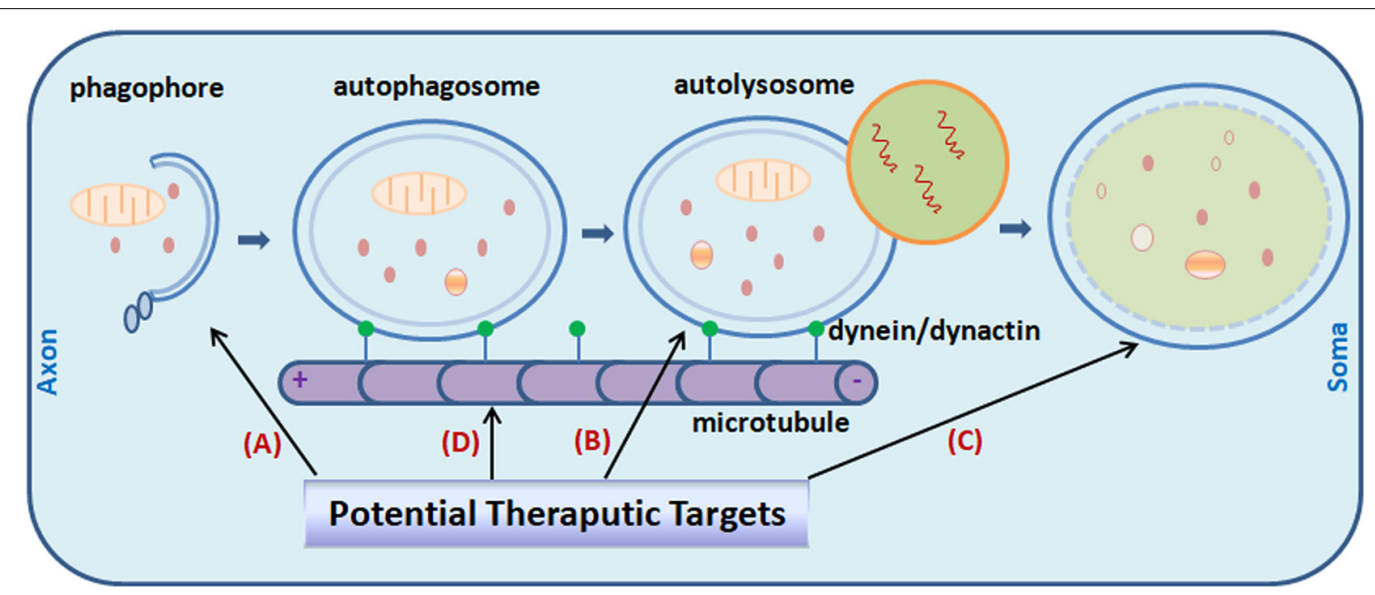

FIGURE 1 | Potential targets for Alzheimer's disease (AD) treatment through the modulating of autophagy. Promoting autophagy induction (A) was originally proposed as an obvious solution to reduce amyloid- $\beta(A \beta)$ aggregates and tau neurofibrillary tangles (NFTs) in the AD brain. It is now realized that the efficiency is context-dependent. Regulating autophagosome-lysosome fusion (B) and enhancing lysosomal function (C) captured more attention recently. Attempts were made to stabilize retrograde transportation of the autophagosomes (D). Combination therapy is also under active investigation currently. Little green dots indicate molecular motor dynein/dynactin. 
2010; Spilman et al., 2010; Majumder et al., 2011). Yet it is noteworthy that the mTOR pathway itself is involved in many other critical cellular functions such as gene translation and cell growth. Toxic side-effects on patients can be induced by long-term inhibition of the mTOR pathway. So, rapamycin is not an ideal drug candidate to be considered for long-term use. Novel specific autophagy inducer is urgently needed for the field. In APP transgenic mice, administration of lentiviral vectors expressing beclin1 lead to induction of autophagy, and reduced both extracellular and intracellular amyloid pathology (Pickford et al., 2008). A point mutation (F121A) reduces the interaction between beclin 1 and its endogenous inhibitor $\mathrm{Bcl} 2$, B-cell lymphoma 2 . Knocking-in of beclin $1_{\mathrm{F} 121 \mathrm{~A}}$ in mice leads to constitutively active autophagy in multiple tissues including brain, even without any autophagy-inducing manipulation. In $\mathrm{AD}$ mouse models, beclin $1_{F 121 A}$-mediated hyperactive autophagy significantly reduces the accumulation of amyloid, and prevents the decline of cognition, and restores the survival rate (Rocchi et al., 2017). Using a gene therapy strategy, Caccamo et al. (2017) showed that cognitive deficits in APP/PS1 mice were rescued by increasing brain p62 expression. Genetic-based approaches may provide more precisely targeted AD therapy. Stimulation of autophagy also reduces neurodegeneration in a mouse model of human tauopathy (Schaeffer et al., 2012).

On the other side, conflicting data arose from recent studies in $\mathrm{AD}$ models suggest that as a generalized treatment strategy for $\mathrm{AD}$, the applicability of induction of autophagy is questionable. Aberrant induction of autophagy may actually lead to elevated $A \beta$ production, as accumulated autophagic vesicles containing active $\gamma$-secretase machinery (Yu et al., 2005; Boland et al., 2008). More and more studies support the notion that the effect of autophagy modulation is context-dependent. Autophagy induction is not always beneficial. Research has shown that a major reservoir for $\mathrm{A} \beta$ production in $\mathrm{AD}$ brain may be autophagosomes ( $\mathrm{Yu}$ et al., 2005). Induction of new autophagosome formation but not accompanied by a parallel autophagic flux increase may actually lead to increased $\mathrm{A} \beta$ production and catabolic contents leaking from AVs (Nixon, 2007). When considering autophagy modulation as a therapy, what is the autophagy defect, when to intervene, and how long/how strong for the modulation, all these should be taken into account. For instance, it has been reported that $A \beta 42-$ induced cell death can be alleviated through inhibition but not stimulation of autophagy (Ling et al., 2009; Wang et al., 2010). So, it appears that the benefit of enhanced induction of autophagy is context-dependent although basal autophagy is required for neuronal survival. This is further demonstrated by the findings that induction of autophagy after the formation of mature tangles and plaques had no effect on cognitive deficits or other AD-like pathology, whereas increasing autophagy induction before the development of AD-like pathology reduced the levels of soluble tau, $A \beta$ and amyloid plaques in $3 \mathrm{xTg}$ $\mathrm{AD}$ mice (Majumder et al., 2011). In addition, conflicting results on the role of autophagy modulation may partially arise from the differences in models. Furthermore, systematic research is necessary for detailed examining the levels of autophagic activity in different cells (neuron vs. glia) in AD as discussed below.

In addition, the accumulation of insoluble $A \beta 42$ over time may be the direct cause of the development of autophagic dysfunction (Steele and Gandy, 2013). In support of this hypothesis, a recent report showed that no conversion to autophagic/lysosomal failure was observed when TgCRND8 mice were treated with scyllo-inositol, an endogenous inositol stereoisomer that is known to inhibit A $\beta 42$ aggregation and fibril formation before the onset of autophagic/lysosomal failure. In contrast, immature AVs and autophagic/lysosomal substrates were significantly accumulated in vehicle-treated TgCRND8 littermates (Lai and McLaurin, 2012).

\section{Enhancing Lysosomal Function}

Recent studies point to impaired lysosomal proteolytic function as the origin of auto-lysosome malfunction in $\mathrm{AD}$ pathogenesis (Yang et al., 2011b). Genetic ablation of cystatin B, an endogenous inhibitor of lysosomal cysteine proteases, in the TgCRND8 AD mouse model with clear defect in proteolytic clearance of autophagic substrates, significantly elevated the lysosomal activity (Figure 1), which leads to enhanced clearance of the autophagic substrates, and obvious alleviation of memory deficits and amyloid pathologies in the animals (Yang et al., 2011a, 2014). Pharmacological compounds with such effects would greatly facilitate research effort in this therapeutic direction (Yang et al., 2017).

\section{Combination Therapy}

Theoretically, it would provide more benefit by simultaneously using two pharmacological autophagy-inducers that act through different regulatory pathways. Indeed, by using the mTOR-independent autophagy enhancer trehalose or lithium and the mTOR-dependent autophagy enhancer rapamycin in combination upregulates autophagy to a greater extent and leads to quicker clearing of protein aggregates than using each alone (Sarkar et al., 2005, 2008). Moreover, using two drugs in combination may enable reduction of the dose of each treatment when compared with either treatment alone, which might greatly reduce the likelihood of adverse effects. In such a scenario, it might be a promising intervention strategy to moderately increase autophagy induction in combination with methods to promote the successful completion of autophagic degradation. However, it is still a big challenge to target the defective lysosomal proteolysis and the autophagy induction at the same time.

In this regard, as it coordinately activates lysosomal biogenesis as well as genes required for autophagosome formation, it seems that transcription factor EB (TFEB) fulfills both of these criteria (Cortes and La Spada, 2019). With its efficacy has already been showed under several neurological conditions, including lysosomal storage disorders (Song et al., 2013), Huntington's disease (HD; Vodicka et al., 2016) and Parkinson's disease (PD; Decressac et al., 2013), it is expected that in the AD context similar benefits may also be achieved. Actually, a study published recently provides the first evidence that TFEB may indeed be beneficial for AD treatment (Xiao et al., 2015). On the other 
hand, pharmacological treatments which improve the catalytic performance of lysosomal enzymes and simultaneously reducing the burden of auto-lysosomal pathway would be another way to tackle the problem of AVs clearance slowdown and disturbed lysosomal function, as exampled by the study of Yang et al. (2017; Figure 1).

\section{Other Strategies}

Alternatively, interventions aiming at reducing the burden to the inappropriate functioning autolysosomal compartments hold some potential as well. For example, preventing $A \beta$ production and oligomerization along with lowering cholesterol may all prove beneficial. In an $\mathrm{AD}$ model, it has been demonstrated that 2-hydroxypropyl-beta-cyclodextrin, a cholesterol-lowering drug, is indeed emerging as a potential useful pharmacological tool (Ren et al., 2016). As for $A \beta$, a recent work implies that useful selective inhibitors of $A \beta$ production could be developed from peptides that disrupt the physical interaction between the APP and PS1 (Das et al., 2003). Finally, as growing evidence suggests that restoring proper endosomal trafficking (recycling) may have a similar effect, another potential strategy to tackle this issue is to develop specific pharmacological modulators of these processes. Two recent studies provided the first proof of concept test through developing pharmacological stabilizer of the retromer sorting complex for $\mathrm{AD}$ treatment (Mecozzi et al., 2014; Young et al., 2018).

\section{DISTURBED AUTOPHAGY IN GLIAL CELLS IN AD}

Astrocytes, microglia, and oligodendrocytes are important components involved in the AD pathogenesis (Dzamba et al., 2016). Under normal conditions, they perform supporting and surveillance functions to neurons in the central nervous system. Engulfing and phagocytosis of extracellular "garbage" like $A \beta$ is vital for neuronal homeostasis. Studies showed that autophagy in glial cells also played a key role in cleaning the microenvironments around neurons (Xue et al., 2014; Pomilio et al., 2016). Disturbing basal autophagy process in glia leads to gliosis and neuroinflammation, which contribute significantly to the development and progression of AD (Herrup et al., 2013; She et al., 2018). Autophagy in glial cells should be taken into account when targeting this process for the treatment of $\mathrm{AD}$.

\section{REFERENCES}

Bakhoum, M. F., Bakhoum, C. Y., Ding, Z., Carlton, S. M., Campbell, G. A., and Jackson, G. R. (2014). Evidence for autophagic gridlock in aging and neurodegeneration. Transl. Res. 164, 1-12. doi: 10.1016/j.trsl.2014.01.016

Barnett, A., and Brewer, G. J. (2011). Autophagy in aging and Alzheimer's disease: pathologic or protective? J. Alzheimers Dis. 25, 385-394. doi: 10.3233/jad-2011101989

Belinson, H., Lev, D., Masliah, E., and Michaelson, D. M. (2008). Activation of the amyloid cascade in apolipoprotein E4 transgenic mice induces lysosomal activation and neurodegeneration resulting in marked cognitive deficits. J. Neurosci. 28, 4690-4701. doi: 10.1523/JNEUROSCI.5633-07.2008

\section{CONCLUSIONS AND FUTURE DIRECTIONS}

The auto-lysosomal function is clearly impaired in $\mathrm{AD}$, which contributes to the accumulation of $A \beta$ plaques and tau NFTs, the two most significant hallmarks of AD. Specific diagnostic methods are urgently needed for accurately identifying and quantifying the autophagic dysfunction in vivo in AD. Currently, the "gold standard" for monitoring autophagy in tissue is direct observation under transmission electron microscopy. It has also been widely used to assess autophagy by Immunohistochemical staining and immunoblotting against autophagy-specific biomarkers such as LC3 (Klionsky et al., 2016). Other approaches include forced expression of GFP-LC3 to detect AVs under the microscope as fluorescent dots, and the use of weakly basic dyes which accumulate in the acidic autophagosome-lysosome compartments (Klionsky et al., 2016). Till now, there is no method to monitor autophagy activity in vivo in a real-time manner.

For a deeper understanding of the dysfunction of autophagic in $\mathrm{AD}$ and for the successful development of therapeutic strategies based on autophagy modulation, it is also very critical to exploring biomarkers that can be applied widely in clinical settings to assess the therapeutic efficiency of autophagy modulation (Chiong, 2018). In addition, as evidence against the druggability of autophagy pathway in the late-stage of the disease, more studies should aim to consider preventive or intervention trials in the early stage of AD (Steele et al., 2013).

\section{AUTHOR CONTRIBUTIONS}

LL conceptualized and designed the study. LL and JL reviewed the literature, wrote the manuscript, and drew the figure; read and approved the final manuscript.

\section{FUNDING}

This work was supported by the National Natural Science Foundation of China (No. 81873775).

\section{ACKNOWLEDGMENTS}

We thank Dr. Hua She for the critical reading of the manuscript and helpful suggestions.

Binder, L. I., Frankfurter, A., and Rebhun, L. I. (1985). The distribution of tau in the mammalian central nervous system. J. Cell Biol. 101, 1371-1378. doi: $10.1083 /$ jcb.101.4.1371

Bingol, B., and Sheng, M. (2011). Deconstruction for reconstruction: the role of proteolysis in neural plasticity and disease. Neuron 69, 22-32. doi: 10.1016/j. neuron.2010.11.006

Blennow, K., De Leon, M. J., and Zetterberg, H. (2006). Alzheimer's disease. Lancet 368, 387-403. doi: 10.1016/S0140-6736(06)69113-7

Boland, B., Kumar, A., Lee, S., Platt, F. M., Wegiel, J., Yu, W. H., et al. (2008). Autophagy induction and autophagosome clearance in neurons: relationship to autophagic pathology in Alzheimer's disease. J. Neurosci. 28, 6926-6937. doi: 10.1523/JNEUROSCI.0800-08.2008 
Boland, B., and Nixon, R. A. (2006). Neuronal macroautophagy: from development to degeneration. Mol. Aspects Med. 27, 503-519. doi: 10.1016/j. mam.2006.08.009

Bordi, M., Berg, M. J., Mohan, P. S., Peterhoff, C. M., Alldred, M. J., Che, S., et al. (2016). Autophagy flux in CA1 neurons of Alzheimer hippocampus: increased induction overburdens failing lysosomes to propel neuritic dystrophy. Autophagy 12, 2467-2483. doi: 10.1080/15548627.2016. 1239003

Caballero, B., Wang, Y., Diaz, A., Tasset, I., Juste, Y. R., Stiller, B., et al. (2018). Interplay of pathogenic forms of human tau with different autophagic pathways. Aging Cell 17:1. doi: 10.1111/acel.12692

Caccamo, A., De Pinto, V., Messina, A., Branca, C., and Oddo, S. (2014). Genetic reduction of mammalian target of rapamycin ameliorates Alzheime's disease-like cognitive and pathological deficits by restoring hippocampal gene expression signature. J. Neurosci. 34, 7988-7998. doi: 10.1523/jneurosci.077714.2014

Caccamo, A., Ferreira, E., Branca, C., and Oddo, S. (2017). p62 improves AD-like pathology by increasing autophagy. Mol. Psychiatry 22, 865-873. doi: $10.1038 / \mathrm{mp} .2016 .139$

Caccamo, A., Magri, A., Medina, D. X., Wisely, E. V., Lopez-Aranda, M. F., Silva, A. J., et al. (2013). mTOR regulates tau phosphorylation and degradation: implications for Alzheimer's disease and other tauopathies. Aging Cell 12, 370-380. doi: 10.1111/acel.12057

Caccamo, A., Majumder, S., Richardson, A., Strong, R., and Oddo, S. (2010). Molecular interplay between mammalian target of rapamycin (mTOR), amyloid- $\beta$, and Tau: effects on cognitive impairments. J. Biol. Chem. 285, 13107-13120. doi: 10.1074/jbc.M110.100420

Cataldo, A. M., Peterhoff, C. M., Schmidt, S. D., Terio, N. B., Duff, K., Beard, M., et al. (2004). Presenilin mutations in familial Alzheimer disease and transgenic mouse models accelerate neuronal lysosomal pathology. J. Neuropathol. Exp. Neurol. 63, 821-830. doi: 10.1093/jnen/63.8.821

Chiong, W. (2018). Challenges in communicating and understanding predictive biomarker imaging for Alzheimer disease. JAMA Neurol. 75, 18-19. doi: 10.1001/jamaneurol.2017.2558

Coen, K., Flannagan, R. S., Baron, S., Carraro-Lacroix, L. R., Wang, D., Vermeire, W., et al. (2012). Lysosomal calcium homeostasis defects, not proton pump defects, cause endo-lysosomal dysfunction in PSEN-deficient cells. J. Cell Biol. 198, 23-35. doi: 10.1083/jcb.201201076

Colacurcio, D. J., Pensalfini, A., Jiang, Y., and Nixon, R. A. (2018). Dysfunction of autophagy and endosomal-lysosomal pathways: roles in pathogenesis of down syndrome and Alzheimer's disease. Free Radic. Biol. Med. 114, 40-51. doi: 10.1016/j.freeradbiomed.2017.10.001

Congdon, E. E. (2018). Sex differences in autophagy contribute to female vulnerability in Alzheimer's disease. Front. Neurosci. 12:372. doi: 10.3389/fnins. 2018.00372

Congdon, E. E., Wu, J. W., Myeku, N., Figueroa, Y. H., Herman, M., Marinec, P. S., et al. (2012). Methylthioninium chloride (methylene blue) induces autophagy and attenuates tauopathy in vitro and in vivo. Autophagy 8, 609-622. doi: 10.4161/auto. 19048

Corradetti, M. N., and Guan, K. L. (2006). Upstream of the mammalian target of rapamycin: do all roads pass through mTOR? Oncogene 25, 6347-6360. doi: 10.1038/sj.onc. 1209885

Cortes, C. J., and La Spada, A. R. (2019). TFEB dysregulation as a driver of autophagy dysfunction in neurodegenerative disease: molecular mechanisms, cellular processes, and emerging therapeutic opportunities. Neurobiol. Dis. 122, 83-93. doi: 10.1016/j.nbd.2018.05.012

Cuervo, A. M., and Wong, E. (2014). Chaperone-mediated autophagy: roles in disease and aging. Cell Res. 24, 92-104. doi: 10.1038/cr.2013.153

Das, C., Berezovska, O., Diehl, T. S., Genet, C., Buldyrev, I., Tsai, J. Y., et al. (2003). Designed helical peptides inhibit an intramembrane protease. J. Am. Chem. Soc. 125, 11794-11795. doi: 10.1021/ja037131v

Decressac, M., Mattsson, B., Weikop, P., Lundblad, M., Jakobsson, J., and Bjorklund, A. (2013). TFEB-mediated autophagy rescues midbrain dopamine neurons from $\alpha$-synuclein toxicity. Proc. Natl. Acad. Sci. U S A 110, E1817-E1826. doi: 10.1073/pnas.1305623110

De Strooper, B., Iwatsubo, T., and Wolfe, M. S. (2012). Presenilins and $\gamma$-secretase: structure, function, and role in Alzheimer disease. Cold Spring Harb. Perspect. Med. 2:a006304. doi: 10.1101/cshperspect.a006304
Dikic, I., and Elazar, Z. (2018). Mechanism and medical implications of mammalian autophagy. Nat. Rev. Mol. Cell Biol. 19, 349-364. doi: 10.1038/s41580-018-0003-4

Dixit, R., Ross, J. L., Goldman, Y. E., and Holzbaur, E. L. (2008). Differential regulation of dynein and kinesin motor proteins by tau. Science 319, 1086-1089. doi: 10.1126/science.1152993

Du, Y., Wooten, M. C., Gearing, M., and Wooten, M. W. (2009a). Age-associated oxidative damage to the 62 promoter: implications for Alzheimer disease. Free Radic. Biol. Med. 46, 492-501. doi: 10.1016/j.freeradbiomed.2008.11.003

Du, Y., Wooten, M. C., and Wooten, M. W. (2009b). Oxidative damage to the promoter region of SQSTM1/p62 is common to neurodegenerative disease. Neurobiol. Dis. 35, 302-310. doi: 10.1016/j.nbd.2009.05.015

Dzamba, D., Harantova, L., Butenko, O., and Anderova, M. (2016). Glial cells-the key elements of Alzheimer's disease. Curr. Alzheimer Res. 13, 894-911. doi: 10.2174/1567205013666160129095924

Esselens, C., Oorschot, V., Baert, V., Raemaekers, T., Spittaels, K., Serneels, L., et al. (2004). Presenilin 1 mediates the turnover of telencephalin in hippocampal neurons via an autophagic degradative pathway. J. Cell Biol. 166, 1041-1054. doi: $10.1083 /$ jcb. 200406060

Hamano, T., Gendron, T. F., Causevic, E., Yen, S. H., Lin, W. L., Isidoro, C., et al. (2008). Autophagic-lysosomal perturbation enhances tau aggregation in transfectants with induced wild-type tau expression. Eur. J. Neurosci. 27, 1119-1130. doi: 10.1111/j.1460-9568.2008.06084.x

Hara, T., Nakamura, K., Matsui, M., Yamamoto, A., Nakahara, Y., SuzukiMigishima, R., et al. (2006). Suppression of basal autophagy in neural cells causes neurodegenerative disease in mice. Nature 441, 885-889. doi: 10.1038 /nature04724

Hardy, J. A., and Higgins, G. A. (1992). Alzheimer's disease: the amyloid cascade hypothesis. Science 256, 184-185. doi: 10.1126/science.1566067

Harris, H., and Rubinsztein, D. C. (2011). Control of autophagy as a therapy for neurodegenerative disease. Nat. Rev. Neurol. 8, 108-117. doi: 10.1038/nrneurol. 2011.200

Hernandez, D., Torres, C. A., Setlik, W., Cebrian, C., Mosharov, E. V., Tang, G., et al. (2012). Regulation of presynaptic neurotransmission by macroautophagy. Neuron 74, 277-284. doi: 10.1016/j.neuron.2012.02.020

Herrup, K., Carrillo, M. C., Schenk, D., Cacace, A., Desanti, S., Fremeau, R., et al. (2013). Beyond amyloid: getting real about nonamyloid targets in Alzheimer's disease. Alzheimers Dement. 9, 452.e1-458.e1. doi: 10.1016/j.jalz.2013. 01.017

Hodson, R. (2018). Alzheimer's disease. Nature 559:S1. doi: 10.1038/d41586-01805717-6

Hollenbeck, P. J. (1993). Products of endocytosis and autophagy are retrieved from axons by regulated retrograde organelle transport. J. Cell Biol. 121, 305-315. doi: $10.1083 /$ jcb.121.2.305

Hung, S. Y., Huang, W. P., Liou, H. C., and Fu, W. M. (2009). Autophagy protects neuron from A $\beta$-induced cytotoxicity. Autophagy 5, 502-510 doi: 10.4161/auto.5.4.8096

Ittner, L. M., Ke, Y. D., Delerue, F., Bi, M., Gladbach, A., Van Eersel, J., et al. (2010). Dendritic function of tau mediates amyloid- $\beta$ toxicity in Alzheimer's disease mouse models. Cell 142, 387-397. doi: 10.1016/j.cell.2010.06.036

Ji, Z. S., Müllendorff, K., Cheng, I. H., Miranda, R. D., Huang, Y., and Mahley, R. W. (2006). Reactivity of apolipoprotein E4 and amyloid $\beta$ peptide: lysosomal stability and neurodegeneration. J. Biol. Chem. 281, 2683-2692. doi: 10.1074/jbc.m506646200

Ji, C., Tang, M., and Johnson, G. V. W. (2017). Assessing the degradation of tau in primary neurons: the role of autophagy. Methods Cell Biol. 141, 229-244. doi: 10.1016/bs.mcb.2017.06.011

Karran, E., Mercken, M., and De Strooper, B. (2011). The amyloid cascade hypothesis for Alzheimer's disease: an appraisal for the development of therapeutics. Nat. Rev. Drug Discov. 10, 698-712. doi: 10.1038/nrd3505

Klionsky, D. J., Abdelmohsen, K., Abe, A., Abedin, M. J., Abeliovich, H., Acevedo Arozena, A., et al. (2016). Guidelines for the use and interpretation of assays for monitoring autophagy (3rd edition). Autophagy 12, 1-222. doi: 10.1080/15548627.2015.1100356

Koike, M., Nakanishi, H., Saftig, P., Ezaki, J., Isahara, K., Ohsawa, Y., et al. (2000). Cathepsin D deficiency induces lysosomal storage with ceroid lipofuscin in mouse CNS neurons. J. Neurosci. 20, 6898-6906. doi: 10.1523/jneurosci.20-1806898.2000 
Komatsu, M., Waguri, S., Chiba, T., Murata, S., Iwata, J., Tanida, I., et al. (2006). Loss of autophagy in the central nervous system causes neurodegeneration in mice. Nature 441, 880-884. doi: 10.1038/nature04723

Lai, A. Y., and McLaurin, J. (2012). Inhibition of amyloid- $\beta$ peptide aggregation rescues the autophagic deficits in the TgCRND8 mouse model of Alzheimer disease. Biochim. Biophys. Acta 1822, 1629-1637. doi: 10.1016/j.bbadis.2012. 07.003

Lee, J. H., Mcbrayer, M. K., Wolfe, D. M., Haslett, L. J., Kumar, A., Sato, Y., et al. (2015). Presenilin 1 maintains lysosomal $\mathrm{Ca}^{2+}$ homeostasis via TRPML1 by regulating vATPase-mediated lysosome acidification. Cell Rep. 12, 1430-1444. doi: 10.1016/j.celrep.2015.07.050

Lee, J. H., Yu, W. H., Kumar, A., Lee, S., Mohan, P. S., Peterhoff, C. M., et al. (2010). Lysosomal proteolysis and autophagy require presenilin 1 and are disrupted by Alzheimer-related PS1 mutations. Cell 141, 1146-1158. doi: 10.1016/j.cell.2010. 05.008

Liang, X. H., Jackson, S., Seaman, M., Brown, K., Kempkes, B., Hibshoosh, H., et al. (1999). Induction of autophagy and inhibition of tumorigenesis by beclin 1. Nature 402, 672-676. doi: 10.1038/45257

Liang, J. H., and Jia, J. P. (2014). Dysfunctional autophagy in Alzheimer's disease: pathogenic roles and therapeutic implications. Neurosci. Bull. 30, 308-316. doi: $10.1007 / \mathrm{s} 12264-013-1418-8$

Lim, F., Hernandez, F., Lucas, J. J., Gomez-Ramos, P., Moran, M. A., and Avila, J. (2001). FTDP-17 mutations in tau transgenic mice provoke lysosomal abnormalities and Tau filaments in forebrain. Mol. Cell. Neurosci. 18, 702-714. doi: $10.1006 /$ mcne. 2001.1051

Lin, W. L., Lewis, J., Yen, S. H., Hutton, M., and Dickson, D. W. (2003). Filamentous tau in oligodendrocytes and astrocytes of transgenic mice expressing the human tau isoform with the P301L mutation. Am. J. Pathol. 162, 213-218. doi: 10.1016/s0002-9440(10)63812-6

Ling, D., Song, H. J., Garza, D., Neufeld, T. P., and Salvaterra, P. M. (2009). A $\beta 42$-induced neurodegeneration via an age-dependent autophagiclysosomal injury in Drosophila. PLoS One 4:e4201. doi: 10.1371/journal.pone.00 04201

Lipinski, M. M., Zheng, B., Lu, T., Yan, Z., Py, B. F., Ng, A., et al. (2010). Genomewide analysis reveals mechanisms modulating autophagy in normal brain aging and in Alzheimer's disease. Proc. Natl. Acad. Sci. U S A 107, 14164-14169. doi: $10.1073 /$ pnas. 1009485107

Maday, S., Wallace, K. E., and Holzbaur, E. L. (2012). Autophagosomes initiate distally and mature during transport toward the cell soma in primary neurons. J. Cell Biol. 196, 407-417. doi: 10.1083/jcb.201106120

Majumder, S., Richardson, A., Strong, R., and Oddo, S. (2011). Inducing autophagy by rapamycin before, but not after, the formation of plaques and tangles ameliorates cognitive deficits. PLoS One 6:e25416. doi: 10.1371/journal. pone.0025416

McGowan, E., Pickford, F., Kim, J., Onstead, L., Eriksen, J., Yu, C., et al. (2005). A 342 is essential for parenchymal and vascular amyloid deposition in mice. Neuron 47, 191-199. doi: 10.1016/j.neuron.2005.06.030

Mecozzi, V. J., Berman, D. E., Simoes, S., Vetanovetz, C., Awal, M. R., Patel, V. M., et al. (2014). Pharmacological chaperones stabilize retromer to limit APP processing. Nat. Chem. Biol. 10, 443-449. doi: 10.1038/nchem bio. 1508

Metaxakis, A., Ploumi, C., and Tavernarakis, N. (2018). Autophagy in age-associated neurodegeneration. Cells 7:E37. doi: 10.3390/cells7050037

Miners, J. S., Baig, S., Palmer, J., Palmer, L. E., Kehoe, P. G., and Love, S. (2008). A $\beta$-degrading enzymes in Alzheimer's disease. Brain Pathol. 18, 240-252. doi: 10.1111/j.1750-3639.2008.00132.x

Mizushima, N., Yamamoto, A., Matsui, M., Yoshimori, T., and Ohsumi, Y. (2004). in vivo analysis of autophagy in response to nutrient starvation using transgenic mice expressing a fluorescent autophagosome marker. Mol. Biol. Cell 15, 1101-1111. doi: 10.1091/mbc.e03-09-0704

Neely, K. M., Green, K. N., and Laferla, F. M. (2011). Presenilin is necessary for efficient proteolysis through the autophagy-lysosome system in a $\gamma$-secretaseindependent manner. J. Neurosci. 31, 2781-2791. doi: 10.1523/jneurosci.515610.2010

Nelson, P. T., Alafuzoff, I., Bigio, E. H., Bouras, C., Braak, H., Cairns, N. J., et al. (2012). Correlation of Alzheimer disease neuropathologic changes with cognitive status: a review of the literature. J. Neuropathol. Exp. Neurol. 71, 362-381. doi: 10.1097/NEN.0b013e31825018f7
Nikoletopoulou, V., and Tavernarakis, N. (2018). Regulation and roles of autophagy at synapses. Trends Cell Biol. 28, 646-661. doi: 10.1016/j.tcb.2018. 03.006

Nilsson, P., and Saido, T. C. (2014). Dual roles for autophagy: degradation and secretion of Alzheimer's disease $\mathrm{A} \beta$ peptide. Bioessays 36, 570-578. doi: 10.1002/bies.201400002

Nilsson, P., Loganathan, K., Sekiguchi, M., Matsuba, Y., Hui, K., Tsubuki, S., et al. (2013). A $\beta$ secretion and plaque formation depend on autophagy. Cell Rep. 5, 61-69. doi: 10.1016/j.celrep.2013.08.042

Nixon, R. A. (2007). Autophagy, amyloidogenesis and Alzheimer disease. J. Cell Sci. 120, 4081-4091. doi: 10.1242/jcs.019265

Nixon, R. A., and Yang, D. S. (2011). Autophagy failure in Alzheimer's disease-locating the primary defect. Neurobiol. Dis. 43, 38-45. doi: 10.1016/j. nbd.2011.01.021

Nixon, R. A., Wegiel, J., Kumar, A., Yu, W. H., Peterhoff, C., Cataldo, A., et al. (2005). Extensive involvement of autophagy in Alzheimer disease: an immuno-electron microscopy study. J. Neuropathol. Exp. Neurol. 64, 113-122. doi: $10.1093 /$ jnen/64.2.113

Noda, T., and Ohsumi, Y. (1998). Tor, a phosphatidylinositol kinase homologue, controls autophagy in yeast. J. Biol. Chem. 273, 3963-3966. doi: 10.1074/jbc. 273.7.3963

Pankiv, S., Clausen, T. H., Lamark, T., Brech, A., Bruun, J. A., Outzen, H., et al. (2007). p62/SQSTM1 binds directly to Atg8/LC3 to facilitate degradation of ubiquitinated protein aggregates by autophagy. J. Biol. Chem. 282, 24131-24145. doi: 10.1074/jbc.M702824200

Pei, J. J., and Hugon, J. (2008). mTOR-dependent signalling in Alzheimer's disease. J. Cell. Mol. Med. 12, 2525-2532. doi: 10.1111/j.1582-4934.2008.00509.x

Peric, A., and Annaert, W. (2015). Early etiology of Alzheimer's disease: tipping the balance toward autophagy or endosomal dysfunction? Acta Neuropathol. 129, 363-381. doi: 10.1007/s00401-014-1379-7

Pickford, F., Masliah, E., Britschgi, M., Lucin, K., Narasimhan, R., Jaeger, P. A., et al. (2008). The autophagy-related protein beclin 1 shows reduced expression in early Alzheimer disease and regulates amyloid $\beta$ accumulation in mice. J. Clin. Invest. 118, 2190-2199. doi: 10.1172/JCI33585

Pomilio, C., Pavia, P., Gorojod, R. M., Vinuesa, A., Alaimo, A., Galvan, V., et al. (2016). Glial alterations from early to late stages in a model of Alzheimer's disease: evidence of autophagy involvement in $\mathrm{A} \beta$ internalization. Hippocampus 26, 194-210. doi: 10.1002/hipo.22503

Ren, B., Jiang, B., Hu, R., Zhang, M., Chen, H., Ma, J., et al. (2016). HP- $\beta$ cyclodextrin as an inhibitor of amyloid- $\beta$ aggregation and toxicity. Phys. Chem. Chem. Phys. 18, 20476-20485. doi: 10.1039/c6cp03582e

Roberson, E. D., Scearce-Levie, K., Palop, J. J., Yan, F., Cheng, I. H., Wu, T., et al. (2007). Reducing endogenous tau ameliorates amyloid $\beta$-induced deficits in an Alzheimer's disease mouse model. Science 316, 750-754. doi: 10.1126/science. 1141736

Rocchi, A., Yamamoto, S., Ting, T., Fan, Y., Sadleir, K., Wang, Y., et al. (2017). A Becn1 mutation mediates hyperactive autophagic sequestration of amyloid oligomers and improved cognition in Alzheimer's disease. PLoS Genet. 13:e1006962. doi: 10.1371/journal.pgen.1006962

Rohn, T. T., Wirawan, E., Brown, R. J., Harris, J. R., Masliah, E., and Vandenabeele, P. (2011). Depletion of Beclin-1 due to proteolytic cleavage by caspases in the Alzheimer's disease brain. Neurobiol. Dis. 43, 68-78. doi: 10.1016/j.nbd.2010.11.003

Sanchez-Varo, R., Trujillo-Estrada, L., Sanchez-Mejias, E., Torres, M., BagliettoVargas, D., Moreno-Gonzalez, I., et al. (2012). Abnormal accumulation of autophagic vesicles correlates with axonal and synaptic pathology in young Alzheimer's mice hippocampus. Acta Neuropathol. 123, 53-70. doi: 10.1007/s00401-011-0896-X

Sarkar, S., Floto, R. A., Berger, Z., Imarisio, S., Cordenier, A., Pasco, M., et al. (2005). Lithium induces autophagy by inhibiting inositol monophosphatase. J. Cell Biol. 170, 1101-1111. doi: 10.1083/jcb.200504035

Sarkar, S., Krishna, G., Imarisio, S., Saiki, S., O’Kane, C. J., and Rubinsztein, D. C. (2008). A rational mechanism for combination treatment of Huntington's disease using lithium and rapamycin. Hum. Mol. Genet. 17, 170-178 doi: $10.1093 / \mathrm{hmg} / \mathrm{ddm} 294$

Schaeffer, V., Lavenir, I., Ozcelik, S., Tolnay, M., Winkler, D. T., and Goedert, M. (2012). Stimulation of autophagy reduces neurodegeneration in a mouse model of human tauopathy. Brain 135, 2169-2177. doi: 10.1093/brain/aws143 
Scheltens, P., Blennow, K., Breteler, M. M., De Strooper, B., Frisoni, G. B., Salloway, S., et al. (2016). Alzheimer's disease. Lancet 388, 505-517. doi: 10.1016/S0140-6736(15)01124-1

She, H., He, Y., Zhao, Y., and Mao, Z. (2018). Release the autophage brake on inflammation: the MAPK14/p38 $\alpha$-ULK1 pedal. Autophagy 14, 1097-1098. doi: 10.1080/15548627.2018.1446626

Shibata, M., Lu, T., Furuya, T., Degterev, A., Mizushima, N., Yoshimori, T., et al. (2006). Regulation of intracellular accumulation of mutant Huntingtin by Beclin 1. J. Biol. Chem. 281, 14474-14485. doi: 10.1074/jbc.m600364200

Shin, J. Y., Park, H. J., Kim, H. N., Oh, S. H., Bae, J. S., Ha, H. J., et al. (2014). Mesenchymal stem cells enhance autophagy and increase $\beta$-amyloid clearance in Alzheimer disease models. Autophagy 10, 32-44. doi: 10.4161/auto.26508

Song, W., Wang, F., Savini, M., Ake, A., Di Ronza, A., Sardiello, M., et al. (2013). TFEB regulates lysosomal proteostasis. Hum. Mol. Genet. 22, 1994-2009. doi: $10.1093 / \mathrm{hmg} / \mathrm{ddt} 052$

Spilman, P., Podlutskaya, N., Hart, M. J., Debnath, J., Gorostiza, O., Bredesen, D., et al. (2010). Inhibition of mTOR by rapamycin abolishes cognitive deficits and reduces amyloid- $\beta$ levels in a mouse model of Alzheimer's disease. PLoS One 5:e9979. doi: 10.1371/journal.pone.0009979

Steele, J. W., Fan, E., Kelahmetoglu, Y., Tian, Y., and Bustos, V. (2013). Modulation of autophagy as a therapeutic target for Alzheimer's disease. Postdoc J. 1, 21-34.

Steele, J. W., and Gandy, S. (2013). Latrepirdine (Dimebon(R)), a potential Alzheimer therapeutic, regulates autophagy and neuropathology in an Alzheimer mouse model. Autophagy 9, 617-618. doi: 10.4161/auto.23487

Suzuki, K., and Terry, R. D. (1967). Fine structural localization of acid phosphatase in senile plaques in Alzheimer's presenile dementia. Acta Neuropathol. 8, 276-284. doi: 10.1007/bf00688828

Tang, Z., Bereczki, E., Zhang, H., Wang, S., Li, C., Ji, X., et al. (2013). Mammalian target of rapamycin (mTor) mediates tau protein dyshomeostasis: implication for Alzheimer disease. J. Biol. Chem. 288, 15556-15570. doi: 10.1074/jbc.m112. 435123

Tang, Y. P., and Gershon, E. S. (2003). Genetic studies in Alzheimer's disease. Dialogues Clin. Neurosci. 5, 17-26.

Tian, Y., Bustos, V., Flajolet, M., and Greengard, P. (2011). A smallmolecule enhancer of autophagy decreases levels of A $\beta$ and APP-CTF via Atg5-dependent autophagy pathway. FASEB J. 25, 1934-1942. doi: 10.1096/fj. $10-175158$

Tomiyama, T., Matsuyama, S., Iso, H., Umeda, T., Takuma, H., Ohnishi, K., et al. (2010). A mouse model of amyloid $\beta$ oligomers: their contribution to synaptic alteration, abnormal tau phosphorylation, glial activation and neuronal loss in vivo. J. Neurosci. 30, 4845-4856. doi: 10.1523/JNEUROSCI.582509.2010

Vassar, R., Bennett, B. D., Babu-Khan, S., Kahn, S., Mendiaz, E. A., Denis, P., et al. (1999). Beta-secretase cleavage of Alzheimer's amyloid precursor protein by the transmembrane aspartic protease BACE. Science 286, 735-741. doi: $10.1126 /$ science.286.5440.735

Vodicka, P., Chase, K., Iuliano, M., Tousley, A., Valentine, D. T., Sapp, E., et al. (2016). Autophagy activation by transcription factor EB (TFEB) in striatum of HDQ175/Q7 mice. J. Huntingtons Dis. 5, 249-260. doi: 10.3233/JHD $-160211$

Wang, H., Ma, J., Tan, Y., Wang, Z., Sheng, C., Chen, S., et al. (2010). Amyloid$\beta 1-42$ induces reactive oxygen species-mediated autophagic cell death in U87 and SH-SY5Y cells. J. Alzheimers Dis. 21, 597-610. doi: 10.3233/JAD-2010091207

Wang, Y., and Mandelkow, E. (2012). Degradation of tau protein by autophagy and proteasomal pathways. Biochem. Soc. Trans. 40, 644-652. doi: 10.1042/BST20120071
Xia, X., Jiang, Q., Mcdermott, J., and Han, J. J. (2018). Aging and Alzheimer's disease: comparison and associations from molecular to system level. Aging Cell 17:e12802. doi: 10.1111/acel.12802

Xiao, Q., Yan, P., Ma, X., Liu, H., Perez, R., Zhu, A., et al. (2015). Neuronaltargeted TFEB accelerates lysosomal degradation of APP, reducing A $\beta$ generation and amyloid plaque pathogenesis. J. Neurosci. 35, 12137-12151. doi: 10.1523/JNEUROSCI.0705-15.2015

Xie, Y., Zhou, B., Lin, M. Y., and Sheng, Z. H. (2015). Progressive endolysosomal deficits impair autophagic clearance beginning at early asymptomatic stages in fALS mice. Autophagy 11, 1934-1936. doi: 10.1080/15548627.2015.1084460

Xue, X., Wang, L. R., Sato, Y., Jiang, Y., Berg, M., Yang, D. S., et al. (2014). Singlewalled carbon nanotubes alleviate autophagic/lysosomal defects in primary glia from a mouse model of Alzheimer's disease. Nano Lett. 14, 5110-5117. doi: $10.1021 / \mathrm{nl} 501839 \mathrm{q}$

Yang, D. S., Stavrides, P., Kumar, A., Jiang, Y., Mohan, P. S., Ohno, M., et al. (2017). Cyclodextrin has conflicting actions on autophagy flux in vivo in brains of normal and Alzheimer model mice. Hum. Mol. Genet. 26, 843-859. doi: $10.1093 / \mathrm{hmg} / \mathrm{ddx} 001$

Yang, D. S., Stavrides, P., Mohan, P. S., Kaushik, S., Kumar, A., Ohno, M., et al. (2011a). Reversal of autophagy dysfunction in the TgCRND8 mouse model of Alzheimer's disease ameliorates amyloid pathologies and memory deficits. Brain 134, 258-277. doi: 10.1093/brain/awq341

Yang, D. S., Stavrides, P., Mohan, P. S., Kaushik, S., Kumar, A., Ohno, M., et al. (2011b). Therapeutic effects of remediating autophagy failure in a mouse model of Alzheimer disease by enhancing lysosomal proteolysis. Autophagy 7, 788-789. doi: 10.4161/auto.7.7.15596

Yang, D. S., Stavrides, P., Saito, M., Kumar, A., Rodriguez-Navarro, J. A. Pawlik, M., et al. (2014). Defective macroautophagic turnover of brain lipids in the TgCRND8 Alzheimer mouse model: prevention by correcting lysosomal proteolytic deficits. Brain 137, 3300-3318. doi: 10.1093/brain/awu278

Young, J. E., Fong, L. K., Frankowski, H., Petsko, G. A., Small, S. A., and Goldstein, L. S. B. (2018). Stabilizing the retromer complex in a human stem cell model of Alzheimer's disease reduces TAU phosphorylation independently of amyloid precursor protein. Stem Cell Reports 10, 1046-1058. doi: 10.1016/j. stemcr.2018.01.031

Yu, W. H., Cuervo, A. M., Kumar, A., Peterhoff, C. M., Schmidt, S. D., Lee, J. H., et al. (2005). Macroautophagy-a novel Beta-amyloid peptidegenerating pathway activated in Alzheimer's disease. J. Cell Biol. 171, 87-98. doi: $10.1083 /$ jcb.200505082

Yue, Z. (2007). Regulation of neuronal autophagy in axon: implication of autophagy in axonal function and dysfunction/degeneration. Autophagy 3, 139-141. doi: 10.4161/auto.3602

Zhang, X., Garbett, K., Veeraraghavalu, K., Wilburn, B., Gilmore, R., Mirnics, K., et al. (2012). A role for presenilins in autophagy revisited: normal acidification of lysosomes in cells lacking PSEN1 and PSEN2. J. Neurosci. 32, 8633-8648. doi: 10.1523/JNEUROSCI.0556-12.2012

Conflict of Interest Statement: The authors declare that the research was conducted in the absence of any commercial or financial relationships that could be construed as a potential conflict of interest.

Copyright (C) 2019 Liu and Li. This is an open-access article distributed under the terms of the Creative Commons Attribution License (CC BY). The use, distribution or reproduction in other forums is permitted, provided the original author(s) and the copyright owner(s) are credited and that the original publication in this journal is cited, in accordance with accepted academic practice. No use, distribution or reproduction is permitted which does not comply with these terms. 